(c) 2003 The American Institute of Physics

To be published in AIP Conference Proceedings of the Space Technology and Applications International Forum (STAIF-2003) Expanding the Frontiers of Space

February 2-6, 2003, Albuquerque, NM

\title{
Update on an Electromagnetic Basis for Inertia, Gravitation, the Principle of Equivalence, Spin and Particle Mass Ratios
}

\author{
Bernard Haisch ${ }^{1}$, Alfonso Rueda ${ }^{2}$, L. J. Nickisch ${ }^{3}$, and Jules Mollere ${ }^{4}$ \\ ${ }^{1}$ Calif. Inst. for Physics \& Astrophysics, 901 Mariners Island Blvd., Ste. 325, San Mateo, CA 94404 \\ ${ }^{2}$ Dept. of Electrical Eng., California State Univ., Long Beach, CA 90840 \\ ${ }^{3}$ Mission Research Corp., Monterey, CA 93940-5776 \\ ${ }^{4}$ Henderson State Univ., Arkadelphia, AR 71999-0001 \\ ${ }^{1}$ haisch@calphysics.org, 650-593-8581, fax: 650-595-4466, <www.calphysics.org>
}

\begin{abstract}
A possible connection between the electromagnetic quantum vacuum and inertia was first published by Haisch, Rueda and Puthoff (1994). If correct, this would imply that mass may be an electromagnetic phenomenon and thus in principle subject to modification, with possible technological implications for propulsion. A multiyear NASA-funded study at the Lockheed Martin Advanced Technology Center further developed this concept, resulting in an independent theoretical validation of the fundamental approach (Rueda and Haisch, 1998ab). Distortion of the quantum vacuum in accelerated reference frames results in a force that appears to account for inertia. We have now shown that the same effect occurs in a region of curved spacetime, thus elucidating the origin of the principle of equivalence (Rueda, Haisch and Tung, 2001). A further connection with general relativity has been drawn by Nickisch and Mollere (2002): zero-point fluctuations give rise to spacetime micro-curvature effects yielding a complementary perspective on the origin of inertia. Numerical simulations of this effect demonstrate the manner in which a massless fundamental particle, e.g. an electron, acquires inertial properties; this also shows the apparent origin of particle spin along lines originally proposed by Schrödinger. Finally, we suggest that the heavier leptons (muon and tau) may be explainable as spatialharmonic resonances of the (fundamental) electron. They would carry the same overall charge, but with the charge now having spatially lobed structure, each lobe of which would respond to higher frequency components of the electromagnetic quantum vacuum, thereby increasing the inertia and thus manifesting a heavier mass.
\end{abstract}

\section{BACKGROUND}

It has been assumed since the late 1960s that an explanation for the origin of mass compatible with the Standard Model of particle physics must involve a postulated Higgs field. The idea is that particles are presumed to acquire the property of mass through interactions with an underlying universal field called the Higgs field, which is carried by the Higgs bosons (as the electromagnetic field is carried by photons). Evidence for the existence of a Higgs field has thus been sought in attempts to create and detect Higgs bosons in colliders by concentration of a sufficiently large amount of energy in particle collisions.

As the Large Electron-Positron Collider (LEP) at CERN approached its final shutdown (to make way for construction of the Large Hadron Collider) in the autumn of 2000, the four LEP detector groups were reporting tentative evidence for a Higgs boson with a mass near $115 \mathrm{GeV} / c^{2}$, at the very limit of the LEP collider (which was already being pushed beyond its design limits). This was a tantalizing possibility, but the enhancement above background of the measurements was less than 3 standard deviations. However further analysis of the LEP data which had been thought to show evidence of the short-lived presence of a Higgs boson failed to demonstrate any convincing signal. This led to the publication of an article in the December 5, 2001 issue of the popular British magazine New Scientist with the headline "No sign of the Higgs boson" together with a strong suggestion that the Higgs does not exist. This anti-Higgs story was widely picked up in the press. However as pointed out by the CERN groups in a dissenting letter also published in that magazine, the mass of the Higgs boson could lie as high as $200 \mathrm{GeV} / c^{2}$. 
Even if the elusive Higgs field is found, this may not shed any light on the property of inertia. As our approach shows, it is possible and potentially fruitful to question whether inertia is simply an innate property of matter, or whether it can be shown to have a separate and specific origin whose mechanism may be altered experimentally (see the discussion of such issues in the monograph by Jammer, 2000). The Standard Model does not address the question of why and how the structure of an elementary particle is able to display inertial effects. For a more extensive discussion of this and related points we refer to Dobyns, Rueda and Haisch (2000) and Haisch, Rueda and Dobyns (2001). The case of gravity is exactly parallel to the situation of inertia as should be expected in light of the principle of equivalence. The proposal that the quantum vacuum (also known in its more restrictive version as the electromagnetic zero-point field, or ZPF) sits at the origin of inertia and gravity, is not only an interesting theoretical proposition in itself but it is also technologically relevant. It opens the possibility to perhaps manipulate inertial and gravitational forces. This would have revolutionary implications for new technologies, especially for space exploration.

In this paper we present a summary of work in progress which indicates how the electromagnetic quantum vacuum interacting with idealized massless particles via the phenomenon of zitterbewegung may explain or at least provide deeper insight into the following fundamental laws and properties of matter:

- origin of inertia (i.e. why does $\mathbf{F}$ equal $m \mathbf{a}$ in Newton's equation of motion?)

- principle of equivalence for inertial and gravitational mass

- de Broglie wavelength of material objects (in particular the electron)

- nature of spin

- mass ratios of fundamental particles (in particular the electron, muon and tau lepton)

Earlier papers on these topics are online at $<$ http://www.calphysics.org/sci_articles.htm $>$.

\section{THE ELECTROMAGNETIC QUANTUM VACUUM (ZPF)}

The Heisenberg uncertainty relation applied to a harmonic oscillator requires that its ground state have a non-zero energy of $h \nu / 2$, since quantum mechanically a particle cannot simultaneously be exactly at the bottom of its potential well and have exactly zero momentum. The quantization of the electromagnetic field in terms of quantum-mechanical operators is found in standard textbooks. As stated by Loudon (1984): "The electromagnetic field is now quantized by the association of a quantum-mechanical harmonic oscillator with each mode $\mathbf{k}$ of the radiation field." Thus there exists the same $h \nu / 2$ zero-point energy expression for each mode of the electromagnetic field as for a mechanical oscillator. Summing up the energy over the modes for all frequencies, directions, and polarization states, one arrives at a zero-point energy density for the electromagnetic fluctuations, and this is the origin of the electromagnetic quantum vacuum. An energy of $h \nu / 2$ per mode of the field characterizes the fluctuations of the quantized radiation field in quantum field theory. In the semi-classical representation of stochastic electrodynamics (SED, see the monographs by de la Peña and Cetto, 1996 and Milonni, 1994) the quantum vacuum is represented by propagating electromagnetic plane waves, $\mathbf{E}^{z p}$ and $\mathbf{B}^{z p}$, of random phase having this average energy, $h \nu / 2$, in each mode.

The volumetric density of modes between frequencies $\nu$ and $\nu+d \nu$ is given by the density of states function $N_{\nu} d \nu=\left(8 \pi \nu^{2} / c^{3}\right) d \nu$. Each state has a minimum $h \nu / 2$ of energy, and using this density of states function and this minimum zero-point energy per state one gets the spectral energy density of the electromagnetic quantum vacuum:

$$
\rho(\nu) d \nu=\frac{8 \pi \nu^{2}}{c^{3}} \frac{h \nu}{2} d \nu
$$

Writing this zero-point radiation together with ordinary blackbody radiation, the energy density is:

$$
\rho(\nu, T) d \nu=\frac{8 \pi \nu^{2}}{c^{3}}\left(\frac{h \nu}{e^{h \nu / k T}-1}+\frac{h \nu}{2}\right) d \nu
$$

The first term (outside the parentheses) represents the mode density, and the terms inside the parentheses are the average energy per mode of thermal radiation at temperature $T$ plus the zero-point energy, $h \nu / 2$. Take away all thermal energy by formally letting $T$ go to zero, and one is still left with the zero-point term. 
The laws of quantum mechanics as applied to electromagnetic radiation force the existence of a background sea of electromagnetic zero-point energy that is traditionally called the electromagnetic quantum vacuum.

It was discovered in the mid-1970's that the quantum vacuum acquires special characteristics when viewed from an accelerating frame. Just as there is an event horizon for a black hole, there is an analogous event horizon for an accelerating reference frame. Similar to radiation from evaporating black holes proposed by Hawking (1974), Unruh (1976) and Davies (1975) determined that a Planck-like radiation component will arise out of the quantum vacuum in a uniformly-accelerating coordinate system having constant proper acceleration a (where $|\mathbf{a}|=a$ ) with what amounts to an effective "temperature"

$$
T_{a}=\frac{\hbar a}{2 \pi c k} .
$$

This "temperature" characterizing Unruh-Davies radiation does not originate in emission from particles undergoing thermal motions. As discussed by Davies, Dray and Manogue (1996):

One of the most curious properties to be discussed in recent years is the prediction that an observer who accelerates in the conventional quantum vacuum of Minkowski space will perceive a bath of radiation, while an inertial observer of course perceives nothing. In the case of linear acceleration, for which there exists an extensive literature, the response of a model particle detector mimics the effect of its being immersed in a bath of thermal radiation (the so-called Unruh effect).

This "heat bath" is a quantum phenomenon. The "temperature" is negligible for most accelerations. Only in the extremely large gravitational fields of black holes or in high-energy particle collisions can this become significant. At the June 2000 meeting of the American Astronomical Society, P. Chen of the Stanford Linear Accelerator Center proposed using an ultra high intensity laser to accelerate electrons violently enough to directly detect Unruh-Davies radiation.

Unruh and Davies treated the electromagnetic quantum vacuum as a scalar field. If a true vectorial approach is considered there appear additional terms beyond the quasi-thermal Unruh-Davies component. For the case of no true external thermal radiation $(T=0)$ but including the acceleration effect $\left(T_{a}\right)$, eqn. (1) becomes (Boyer 1980)

$$
\rho\left(\nu, T_{a}\right) d \nu=\frac{8 \pi \nu^{2}}{c^{3}}\left[1+\left(\frac{a}{2 \pi c \nu}\right)^{2}\right]\left[\frac{h \nu}{2}+\frac{h \nu}{e^{h \nu / k T_{a}}-1}\right] d \nu .
$$

While these acceleration-dependent terms do not show any spatial asymmetry in the expression for the spectral energy density, an asymmetry does appear when the momentum flux of this radiation is calculated, resulting in a non-zero flux. This appears to be the process underlying inertial and gravitational forces.

\section{ZITTERBEWEGUNG}

In his study of the coordinate operator in the Dirac equation, Schrödinger $(1930,1931)$ discovered microscopic oscillatory motion at the speed of light, which he called zitterbewegung. While Dirac argued that such motion does not violate relativity or quantum theory (see Dirac, 1958), from a classical particle point of view, these speed of light motions would seem to imply masslessness of the particle. Dirac theory also describes particle spin, and Schrödinger considered spin to be an orbital angular momentum that is a consequence of the vacuum fields. This view of spin was explored further by Huang (1952) and Barut and Zanghi (1984).

We take the view presented in the monograph by de la Peña and Cetto (1996) that zitterbewegung is the result of electromagnetic quantum vacuum fluctuations acting upon a fundamentally point-like massless charged particle. We discuss below how such a particle constantly undergoing transverse changes in direction due to these fluctuations would manifest the property of inertia, hence appearing to possess the property of mass. The spatially averaged size of a point-like particle undergoing such perturbations corresponds to the Compton radius (see MacGregor, 1992), thus showing one of several connections between quantum properties and zitterbewegung (Hestenes, 1990). Spin and the de Broglie wavelength can also be understood from this perspective. 


\section{THE LORENTZ FORCE APPOACH TO INERTIA (HRP)}

In the paper "Inertia as a zero-point field Lorentz force" Haisch, Rueda and Puthoff (HRP, 1994) assumed that a fundamental particle (such as an electron) could be treated as a two-dimensional Planck oscillator driven the by electric components $\left(\mathbf{E}^{z p}\right)$ of the ZPF to oscillate in the $x y$-plane. They then examined the effects of the magnetic components $\left(\mathbf{B}^{z p}\right)$ of the ZPF on the Planck oscillator under the condition of constant acceleration in the $z$-direction. The result was that the Lorentz force due to $\mathbf{B}^{z p}$ fluctuations proved to be proportional to the acceleration of the Planck oscillator, thus suggesting its interpretation as the reaction force due to inertia.

\section{THE POYNTING VECTOR APPROACH TO INERTIA (RH)}

The approach by Rueda and Haisch $(\mathrm{RH})$ relies on making standard transformations of the $\mathbf{E}^{z p}$ and $\mathbf{B}^{z p}$ from a stationary to an accelerated coordinate system. In a stationary or uniformly-moving frame the $\mathbf{E}^{z p}$ and $\mathbf{B}^{z p}$ constitute an isotropic radiation pattern. In an accelerated frame the radiation pattern acquires asymmetries. There turns out to be a non-zero Poynting vector in any accelerated frame, and this carries a non-zero net flux of electromagnetic momentum. The scattering of this momentum flux generates a reaction force, $\mathbf{F}_{r}$. RH found an invariant scalar with the dimension of mass describing the resistance to acceleration resulting from this process. We interpret this scalar as the inertial mass,

$$
m_{i}=\frac{V_{0}}{c^{2}} \int \eta(\nu) \rho_{z p}(\nu) d \nu,
$$

where $\rho_{z p}$ is the well known spectral energy density of the electromagnetic quantum vacuum of eqn. (1). In other words, the amount of electromagnetic zero point energy instantaneously transiting through an object of volume $V_{0}$ and interacting with the quarks and electrons in that object is what constitutes the inertial mass of that object. It is change in the momentum of the radiation field that creates the resistance to acceleration usually attributed to the inertia of an object.

Indeed, not only does the ordinary form of Newton's second law, $\mathbf{F}=m_{i} \mathbf{a}$, emerge from this analysis, but one can also obtain the relativistic form of the second law:

$$
\mathcal{F}=\frac{d \mathcal{P}}{d \tau}=\frac{d}{d \tau}\left(\gamma_{\tau} m_{i} c, \mathbf{p}\right)
$$

The origin of inertia, in this picture, becomes remarkably intuitive. Any material object resists acceleration because the acceleration produces a perceived flux of radiation in the opposite direction that scatters within the object and thereby pushes against the accelerating agent. Inertia in the present model appears as a kind of acceleration-dependent electromagnetic quantum vacuum drag force acting upon electromagneticallyinteracting elementary particles (electrons and quarks). The relativistic law for "mass" transformation that is, the formula describing how the inertia of a body has been calculated to change according to an observer's relative motion - is automatically satisfied in this view, because the correct relativistic form of the reaction force is derived, as shown in eqn. (6).

\section{THE NEW CONNECTIVITY APPROACH}

Both of the approaches above (together called the RHP approach for convenience) assume classical electrodynamics operating in flat spacetime. Einstein's field equations for general relativity (GR)

$$
G_{\mu \nu}=8 \pi T_{\mu \nu}
$$

describe how curved spacetime geometry $\left(G_{\mu \nu}\right)$ is produced by the presence of matter or energy as described by the energy-momentum tensor $\left(T_{\mu \nu}\right)$. Nickisch and Mollere have considered the possibility that electromagnetic fields, including that of the zero-point fluctuations, can be treated as a distortion in the spacetime of the charge. A massless charge would behave like a photon, following a null geodesic, but in a spacetime 
defined by electromagnetic fields. A photon, in the absence of any energy or matter other than the zero-point fluctuations, will follow an unperturbed flat spacetime trajectory. However unlike the spacetime of a photon, the spacetime of the massless charge is defined by the distortions of the zero-point fluctuations, producing a geodesic description of zitterbewegung. Additional electromagnetic fields may produce a non-zero-mean drift of the zitterbewegung that is also accounted for in the geodesic motion. These non-zero-mean effects "accumulate" into a stretching of the particle's spacetime, and this stretching is perceived by external observers to be inertia.

In the Nickisch-Mollere "Connectivity" theory, the ZPF defines a curvature in the particle's spacetime. The metric describing this curvature implies a transformation to the viewpoint of an observer who assumes spacetime is flat (the Connective transformation). Application of the Connective transformation produces the usual effects of inertia when observed in Minkowski (flat) spacetime, including hyperbolic motion in a static electric field (above the vacuum) and uniform motion following an impulse. The motion of the massless charge is a helical motion that can be equated to the particle spin of quantum theory. This spin has the properties expected from quantum theory, being undetermined until "measured" by applying a field, and then being found in either a spin up or spin down state.

In Connectivity it is assumed that the equation of motion of a massless charge is that of a geodesic in a spacetime whose curvature is defined by the electromagnetic fields encountered by the particle. Since the charge is assumed to be massless and moving at the speed of light (following a "null" curve, in the terminology of relativity), proper time cannot be used as the affine parameter of the geodesic (proper time intervals vanish for null geodesics). However, normal time serves well as an affine parameter. The equation of the geodesic is therefore taken to be

$$
\frac{d p^{\mu}}{d t}+\frac{1}{m_{*}} \Gamma_{\nu \rho}^{\mu} p^{\nu} p^{\rho}=0
$$

where

$$
p^{\mu}=m_{*} c n^{\mu},
$$

and

$$
n^{\mu}=\left(n^{0}, \mathbf{n}\right) .
$$

Here $p^{\mu}$ is the four-momentum of the charge $q, c$ is the speed of light, $F^{\mu \nu}$ is the electromagnetic field tensor of the impressed fields including the ZPF, and $n^{\mu}$ is the direction vector of the particle motion. The mass parameter $m_{*}$ has the dimensions of mass, but it is not mass; particles described by eqn. (8) move at the speed of light. $\Gamma_{\nu \rho}^{\mu}$ are the Christoffel symbols of the second kind. The connection terms (the terms containing the Christoffel symbols) are equated with the Lorentz force, thus showing the connection between this approach and that of HRP. That is,

$$
\Gamma_{\nu \rho}^{\mu} p^{\nu} p^{\rho}=-\frac{q}{c} F_{\nu}^{\mu} p^{\nu} n^{0}
$$

These equations can be solved for the metric $g_{\mu \nu}$ of the particle's spacetime, though not uniquely. The equations (11) actually define a class of metrics. Further constraints are required to select a particular solution from this class. In particular, the geodesic in the particle spacetime should be a null curve as expected for a massless object. One interesting aspect of the metrics derived from eqn. (11) is that they turn out to depend on the particle history through time integrations. Since each particle experiences its own history of encountered fields (including zero-point fluctuations), this means that the local spacetime distortion observed by a particle is unique to it; two particles at the same place and time will, in general, see spacetime stretched in a somewhat different way. This, in Connectivity, is how two identical massless charges can have different momenta.

Using eqn. (11), the geodesic equation (8) can be written as,

$$
\frac{d n^{j}}{d t}=\frac{q}{m_{*} c}\left[F_{\nu}^{j} n^{\nu} n^{0}-F_{\nu}^{0} n^{\nu} n^{j}\right]+\frac{n^{j}}{n^{0}} \frac{d n^{0}}{d t},
$$




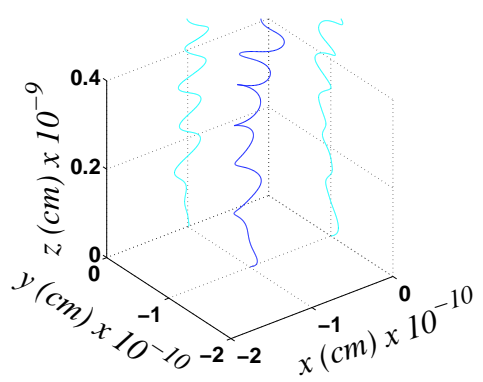

FIGURE 1. Massless Charge Trajectory in a Uniform Electric Field plus the ZPF, Showing Spin-like Orbital Motion and its Projections onto the $x z$ and $y z$ Planes.

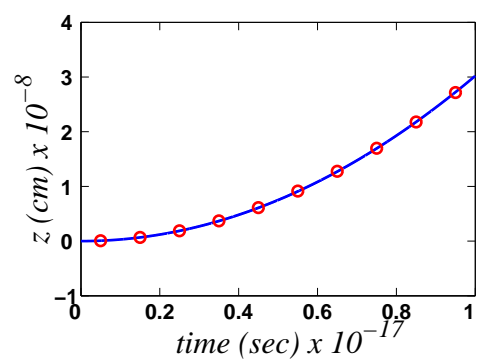

FIGURE 2. Massless Charge Motion in a Uniform Electric Field plus the ZPF, Obtained Using Connectivity (solid curve), compared to the Hyperbolic Motion of a Massive Charge in Special Relativity (circles).

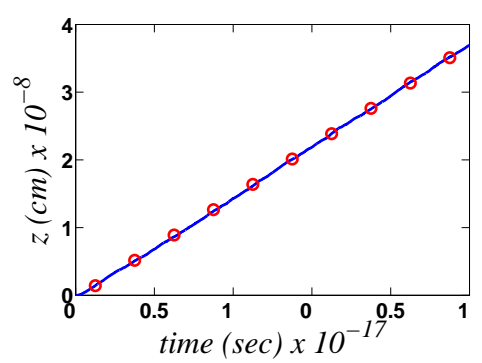

FIGURE 3. Massless Charge Motion in the ZPF Following an Impulse, Obtained Using Connectivity (solid curve), Compared to Constant Speed Motion (circles).

$$
\frac{d m_{*}}{d t}=\frac{q}{c} F_{\nu}^{0} n^{\nu}-\frac{m_{*}}{n^{0}} \frac{d n^{0}}{d t} .
$$

In general $n^{0}$ does not retain a value of unity, but changes in a way that preserves the null curve property,

$$
g_{\mu \nu} p^{\mu} p^{\nu}=0
$$

Note that eqn. (13), which is the zeroth equation of eqn. (8), is an equation for the parameter $m_{*}$. Thus $m_{*}$ is not a constant, but rather varies in response to applied forces. The effect is to introduce time dilation (or Doppler shifting) in the energy-momentum four vector analogous to the gravitational redshift of GR.

Eqns. (8-14) describe the motion of a massless charge in response to impressed electromagnetic fields. The charge moves at a constant speed (the speed of light) with a changing direction given by eqn. (12). When the impressed fields include the ZPF, this motion may be regarded as Schrödinger's zitterbewegung. When a field above the vacuum is applied, the charge will be observed to drift in a preferred direction in its zitterbewegung wander. This is illustrated in Figure 1, which shows the trajectory of a massless charge computed from eqn. (8) using one of the metrics from the class of metrics implied by eqn. (11). The electromagnetic fields influencing the motion of the charge are a random realization of the ZPF with a superimposed uniform electric field in the vertical direction (the driving field). Note that the charge drifts upward in response to the driving field. We see that the ZPF drives the charge in a pseudo-helical motion as in Schrödinger's orbital angular momentum explanation for spin.

The metric $g_{\mu \nu}$ implies a transformation to Minkowski (flat) spacetime, the so-called Connective transformation. The transformation $C_{\mu}^{\nu}$ from the particle's spacetime to Minkowski spacetime is related to the metric $g_{\mu \nu}$ by $g_{\mu \nu}=C_{\mu}^{\rho} C_{\nu}^{\sigma} \eta_{\rho \sigma}$, or

$$
g=C \cdot \eta \cdot \tilde{C}
$$

where $\tilde{C}$ is the transpose of $C$ and $\eta$ is the flat spacetime metric.

It is the application of the Connective transformation that allows one to view the particle trajectory in Minkowski spacetime, and here the effects of inertia appear. Two of the simplest manifestations of inertia are hyperbolic motion of a charge in the presence of a uniform electric field and uniform motion following an impulse. Figure 2 displays the component of the motion of a charge in the direction of a uniform electric field applied above the ZPF, obtained by solving eqn. (8) and applying the Connective transformation to view the result in Minkowski spacetime. The solid curve is the result of the Connectivity simulation. The circles lie on the hyperbola defined by a massive charge undergoing uniform acceleration in special relativity. The agreement is striking. Viewed in Minkowski spacetime, the massless charge is seen to accelerate hyperbolically as though it had inertia. Figure 3 is a similar depiction for the case in which an impulse has been applied to the charge, and the charge is observed in Minkowski spacetime to continue in uniform motion following the impulse. Here the circles lie on a straight line, indicating that the particle travels at a constant speed 

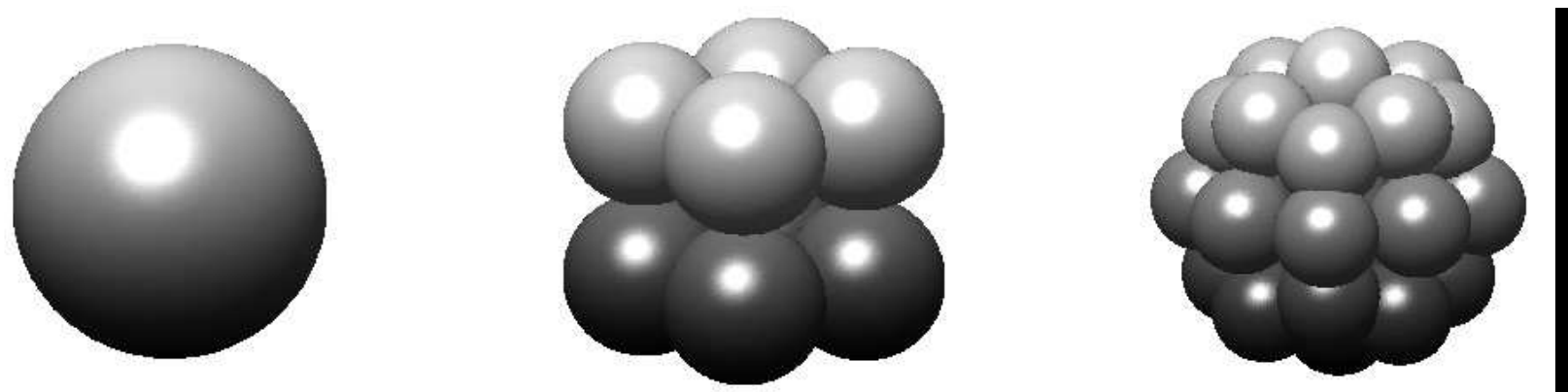

FIGURE 4. Notional Depiction of Charge Sub-volumes that Yield Lepton Mass Spectrum as Harmonic Structures.

following the impulse. Note that it is the average motion of the charge that moves uniformly. Deviations about the average motion are apparent and are, in fact, zitterbewegung driven by the ZPF. The average motion defines a timelike curve, as expected of a massive particle. This dynamic is well-modelled by a moving center of mass with speed-of-light motions of the charge center about it, and is therefore consistent with the successful "displaced charge center" model that is often invoked to describe the zitterbewegung of classical charges (see, e.g., Rueda, 1993). The beauty of Connectivity is that the full dynamics of the displaced charge center model is obtained without having to assume particle structure, or rather, the particle structure of that model is obtained in a natural way.

When the forces acting on the charge are the Lorentz forces due to the electromagnetic vacuum fields (the ZPF), these drive the charge in zitterbewegung motion at the speed of light, in agreement with the speed-oflight eigenvalues of the Dirac theory. When the charge moves with a large average velocity in some direction, the zitterbewegung motion extends to a quasi-helical motion that may be the basis of particle spin. This spin is undetermined until "measured" by applying a field that aligns the zitterbewegung into helical motion, which will either be oriented with positive or negative helicity (spin up or spin down, see Figure 1).

The RHP theory of inertia has the implication that potentially all mass is due to interaction of bare massless charges with the vacuum fields, where "charge" is understood in the generalized sense as the charge associated with any fundamental vacuum field. It has recently been argued that since the effects of the ZPF on a massless particle moving at the speed of light can only involve transverse forces, no work can be done, hence no energy transferred from the ZPF to the particle, thus contradicting the proposed RHP inertia-generating mechanism (Ibison 2001). This problem is resolved in the Connectivity approach to inertia. Electromagnetic fields are assumed to describe the curvature of spacetime, and massless charges simply follow geodesic motion in this curved spacetime. When the view is transformed to the flat spacetime assumption, inertial forces appear.

Since the interpretation of Connectivity is that electromagnetic fields define a curvature of spacetime, it may be possible to show that radiation is related to the distortion of spacetime required to connect the views of different frames. This should then have implications for the problem of the stability of atoms. Furthermore, these distortions of spacetime are presumably no different from that described by the usual gravitational form of GR; it is simply a matter of scale. The ZPF represents spacetime distortions on a very fine scale. However, if there are many charges in a localized place (say in a star), then their presence (as boundary conditions for the $\mathrm{ZPF}$ and including their collective $\mathrm{ZPF}$-induced radiation) changes the normally isotropic ZPF to an EM-field distribution yielding a broad overall spacetime curvature in that region. This broad curvature may in fact be the spacetime curvature usually assigned to gravity in GR, thereby providing the potential to relate gravitational and inertial mass.

We conclude with a conjecture that, while speculative, is at least worthy of further study. The RHP theory implies that the effective inertial mass of a charge scales with either the third power of a resonance frequency or the fourth power of a cutoff frequency for the charge's interaction with the ZPF. If this cutoff is associated with a finite charge distribution size, then it is reasonable to expect that the charge distribution itself may be capable of exhibiting a harmonic mode structure. Now imagine that for some reason the first strongly observable harmonic structure has the charge distribution exhibiting a two-lobe structure in each of the three spatial dimensions, with the second strongly observable harmonic exhibiting a three-lobe structure in each of the three dimensions. This is shown notionally in Figure 4. The number of charge sub-volumes so 
generated scales as the cube of the cutoff frequency of the fundamental. All told, then, the masses of the higher states will scale as the sixth or the seventh power of a resonance or a cutoff frequency respectively of the fundamental since each sub-lobe will be responsive to proportionately higher frequencies of the ZPF (i.e., a sub-lobe with half the size of the fundamental charge distribution size can be translated by wavelengths twice as small as the fundamental charge distribution size). When this conjecture is applied to the lepton mass spectrum, we find for the masses of the muon and tauon relative to the electron that $\left(m_{\mu} / m_{e}\right)^{1 / 7}=2.1$, $\left(m_{\tau} / m_{e}\right)^{1 / 7}=3.2$ for the case of a cutoff frequency or $\left(m_{\mu} / m_{e}\right)^{1 / 6}=2.4,\left(m_{\tau} / m_{e}\right)^{1 / 6}=3.9$ for the case of a resonance. Form factors associated with the actual shapes of the harmonic charge structures could easily account for the small deviations of these results from integer values. The spin of each of these states is associated with the zitterbewegung transport of the entire body as a whole, so the spin of each of the leptons will be the same. Although this mass relation is only conjecture, it provides an indication of the potential for a vacuum field origin of inertia to explain the mass spectra of elementary particles.

\section{ORIGIN OF WEIGHT AND THE WEAK EQUIVALENCE PRINCIPLE}

Einstein introduced the local Lorentz invariance (LLI) principle in order to pass from special relativity to GR. It is possible to use this principle immediately to extend the results of the quantum vacuum inertia hypothesis to gravitation (details discussed in Rueda, Haisch and Tung, 2001).

The idea behind the LLI principle is embodied in the Einstein elevator thought experiment. He proposed that a freely-falling elevator in a gravitational field is equivalent to one that is not accelerating and is far from any gravitating body. Physics experiments would yield the same results in either elevator, and therefore a freely-falling coordinate frame in a gravitational field is the same as an inertial Lorentz frame. (This is rigorously only true for a "small elevator" since a gravitational field around a planet, say, must be radial, hence there are inevitably tidal forces which would not be the case for an ideal acceleration.) The device Einstein used to develop GR was to invoke an infinite set of such freely falling frames. In each such frame, the laws of physics are those of special relativity. The additional features of GR emerge by comparing the properties of measurements made in freely-falling Lorentz frames "dropped" one after the other.

This approach of Einstein is both elegant and powerful. The LLI principle immediately tells us that an object accelerating through the electromagnetic quantum vacuum is equivalent to an object held fixed in a gravitational field while the electromagnetic quantum vacuum is effectively accelerating (falling) past it. The prediction of GR that light rays deviate from straight-line propagation in the presence of a gravitating

body translates into acceleration (falling) of the electromagnetic quantum vacuum. An object accelerating through the electromagnetic quantum vacuum experiences a flux which causes the inertia reaction force. A fixed object past which the electromagnetic quantum vacuum is accelerating, following the laws of GR, experiences the same flux and the resulting force is what we call weight. That is why $m_{g}=m_{i}$ and is the basis of the weak equivalence principle.

\section{CONCLUSIONS}

It appears that a simple model of a particle as an electromagnrtic quantum vacuum-driven oscillating charge with a resonance at its Compton frequency may simultaneously offer insight into the nature of inertial and gravitational mass, the origin of the de Broglie wavelength and spin, and possibly particle mass ratios. For a recent popular-level discussion of our appoach to the nature of mass see the article by Chown (2001) in New Scientist. Numerous papers are also online at $<$ http://www.calphysics.org/sci_articles.htm] $>$.

\section{APPENDIX: INERTIA AND THE DE BROGLIE WAVELENGTH}

Four-momentum is defined as

$$
\mathbf{P}=\left(\frac{E}{c}, \mathbf{p}\right)=\left(\gamma m_{0} c, \mathbf{p}\right)=\left(\gamma m_{0} c, \gamma m_{0} \mathbf{v}\right)
$$


where $|\mathbf{P}|=m_{0} c$ and $E=\gamma m_{0} c^{2}$. The Einstein-de Broglie relation defines the Compton frequency $h \nu_{C}=$ $m_{o} c^{2}$ for an object of rest mass $m_{0}$, and if we make the de Broglie assumption that the momentum-wave number relation for light also characterizes matter then $\mathbf{p}=\hbar \mathbf{k}_{B}$ where $\mathbf{k}_{B}=2 \pi\left(\lambda_{B, 1}^{-1}, \lambda_{B, 2}^{-1}, \lambda_{B, 3}^{-1}\right)$. We thus write

$$
\frac{\mathbf{P}}{\hbar}=\left(\frac{2 \pi \gamma \nu_{C}}{c}, \mathbf{k}_{B}\right)=2 \pi\left(\frac{\gamma}{\lambda_{C}}, \frac{1}{\lambda_{B, 1}}, \frac{1}{\lambda_{B, 2}}, \frac{1}{\lambda_{B, 3}}\right)
$$

and from this obtain the relationship

$$
\lambda_{B}=\frac{c}{\gamma v} \lambda_{C}
$$

between the Compton wavelength, $\lambda_{C}$, and the de Broglie wavelength, $\lambda_{B}$. For a stationary object $\lambda_{B}$ is infinite, and the de Broglie wavelength decreases in inverse proportion to the momentum.

Eqn. (5) is very suggestive that quantum vacuum-elementary particle interaction involves a resonance at the Compton frequency. de Broglie proposed that an elementary particle is associated with a localized wave whose frequency is the Compton frequency. As summarized by Hunter (1996): “... what we regard as the (inertial) mass of the particle is, according to de Broglie's proposal, simply the vibrational energy (divided by $c^{2}$ ) of a localized oscillating field (most likely the electromagnetic field). From this standpoint inertial mass is not an elementary property of a particle, but rather a property derived from the localized oscillation of the (electromagnetic) field. de Broglie described this equivalence between mass and the energy of oscillational motion... as 'une grande loi de la Nature' (a great law of nature)."

This perspective is consistent with the proposition that inertial mass, $m_{i}$, may be a coupling parameter between electromagnetically interacting particles and the quantum vacuum. Although de Broglie assumed that his wave at the Compton frequency originates in the particle itself (due to some intrinsic oscillation or circulation of charge perhaps) there is an alternative interpretation discussed in some detail by de la Peña and Cetto that a particle "is tuned to a wave originating in the high-frequency modes of the zero-point background field." The de Broglie oscillation would thus be due to a resonant interaction with the quantum vacuum, presumably the same resonance that is responsible for creating a contribution to inertial mass as in eqn. (5). In other words, the electromagnetic quantum vacuum would be driving this $\nu_{C}$ oscillation.

We therefore suggest that an elementary charge driven to oscillate at the Compton frequency, $\nu_{C}$, by the quantum vacuum may be the physical basis of the $\eta(\nu)$ scattering parameter in eqn. (5). For the case of the electron, this would imply that $\eta(\nu)$ is a sharply-peaked resonance at the frequency, expressed in terms of energy, $h \nu_{C}=512 \mathrm{keV}$. The inertial mass of the electron would physically be the reaction force due to resonance scattering of the electromagnetic quantum vacuum radiation at that frequency.

This leads to a surprising corollary. It has been shown that as viewed from a laboratory frame, a standing wave at the Compton frequency in the electron frame transforms into a traveling wave having the de Broglie wavelength for a moving electron. (Hunter, 1996; de la Peña and Cetto, 1996; Kracklauer, 1992; Haisch and Rueda, 2000) The wave nature of the moving electron (as measured in the Davisson-Germer experiment, for example) would be basically due to Doppler shifts associated with its Einstein-de Broglie resonance at the Compton frequency. A simplified heuristic model shows this, and a detailed treatment showing the same result may be found in de la Peña and Cetto. Represent a quantum vacuum-like driving force field as two waves having the Compton frequency $\omega_{C}=2 \pi \nu_{C}$ travelling in equal and opposite directions, $\pm \hat{x}$. The amplitude of the combined oppositely-moving waves acting upon an electron will be

$$
\phi=\phi_{+}+\phi_{-}=2 \cos \omega_{C} t \cos k_{C} x
$$

But now assume an electron is moving with velocity $v$ in the $+x$-direction. The wave responsible for driving the resonant oscillation impinging on the electron from the front will be the wave seen in the laboratory frame to have frequency $\omega_{-}=\gamma \omega_{C}(1-v / c)$, i.e. it is the wave below the Compton frequency in the laboratory that for the electron is Doppler shifted up to the $\omega_{C}$ resonance. Similarly the zero-point wave responsible for driving the electron resonant oscillation impinging on the electron from the rear will have a laboratory frequency $\omega_{+}=\gamma \omega_{C}(1+v / c)$ which is Doppler shifted down to $\omega_{C}$ for the electron. The same transformations apply to the wave numbers, $k_{+}$and $k_{-}$. The Lorentz invariance of the electromagnetic quantum vacuum 
spectrum ensures that regardless of the electron's (unaccelerated) motion the up- and down-shifting of the laboratory-frame spectral energy density will always yield a standing wave in the electron's frame.

It can be shown that the superposition of these two oppositely-moving, Doppler-shifted waves is

$$
\phi^{\prime}=\phi_{+}^{\prime}+\phi_{-}^{\prime}=2 \cos \left(\gamma \omega_{C} t-k_{B} x\right) \cos \left(\omega_{B} t-\gamma k_{C} x\right)
$$

Observe that for fixed $x$, the rapidly oscillating "carrier" of frequency $\gamma \omega_{C}$ is modulated by the slowly varying envelope function in frequency $\omega_{B}$. And vice versa observe that at a given $t$ the "carrier" in space appears to have a relatively large wave number $\gamma k_{C}$ which is modulated by the envelope of much smaller wave number $k_{B}$. Hence both timewise at a fixed point in space and spacewise at a given time, there appears a carrier that is modulated by a much broader wave of dimension corresponding to the de Broglie time $t_{B}=2 \pi / \omega_{B}$, or equivalently, the de Broglie wavelength $\lambda_{B}=2 \pi / k_{B}$.

de la Peña and Cetto (1996) generalize this to include quantum vacuum radiation from all other directions and conclude: "The foregoing discussion assigns a physical meaning to de Broglie's wave: it is the modulation of the wave formed by the Lorentz-transformed, Doppler-shifted superposition of the whole set of random stationary electromagnetic waves of frequency $\omega_{C}$ with which the electron interacts selectively." Another way of looking at the spatial modulation is in terms of the wave function: the spatial modulation of eqn. (A5) is exactly the $e^{i p x / \hbar}$ wave function of a freely moving particle satisfying the Schrödinger equation (Hunter, 1996). In such a view the quantum wave function of a moving free particle becomes a "beat frequency" produced by the relative motion of the observer with respect to the particle and its oscillating charge.

\section{REFERENCES}

Barut A. O. and Zanghi, N., Phys. Rev. Lett. 52, 2009 (1984).

Boyer, T. H., Phys. Rev. D 21, 2137 (1980).

Chown, M., New Scientist, No. 2276, 3 Feb. (2001).

Davies, P. C. W., J. Phys. A 8, 609 (1975).

de la Peña, L. and Cetto, A.M., The Quantum Dice: An Introduction to Stochastic Electrodynamics, (Kluwer Acad. Publ.), (1996).

Davies, P. C. W., Dray, T. and Manogue, C. A., Phys. Rev. D 53, 4382 (1996).

Dirac, P. A .M.The Principles of Quantum Mechanics, (Clarendon, Oxford), 4th edition, p. 262 (1958).

Dobyns, Y., Rueda, A. and B. Haisch, Found. Phys., 30, No.1, 59, (2000)

Haisch, B., Rueda, A. and Dobyns, Y., Ann. Phys. (Leipzig), 10, No. 5, 393-414, (2001).

Haisch, B. and Rueda, A. Phys. Lett. A 268, 224 (2000).

Haisch, B., Rueda, A. and Puthoff, H.E. (HRP), Phys. Rev. A 49, 678 (1994).

Hawking, S. Nature 248, 30 (1974).

Hestenes, D., Found. Phys., 20, No. 10, 1213 (1990).

Huang, K. Am. J. Physics 20, 479 (1952).

Hunter, G. in The Present Status of the Quantum Theory of Light, S. Jeffers et al. (eds.), (Kluwer Acad. Publ.), chap. $12(1996)$

Ibison, M., www.arxiv.org/abs/physics/0106080 (2001).

Jammer, M. Concepts of Mass in Contemporary Physics and Philosopy, Princeton Univ. Press (2000).

Kracklauer, A. F., Physics Essays 5, 226 (1992).

Loudon, R., The Quantum Theory of Light, chap. 1, (Oxford: Clarendon Press) (1983).

MacGregor, M., The Enigmatic Electron, Kluwer, (1992).

Milonni, P.W. The Quantum Vacuum, Academic Press (1994).

Nickisch, L. J. and J. Mollere, www.arxiv.org/abs/physics/0205086 (2002).

Rueda, A. Found. Phys. Lett., 6, no. 1, 75 and no. 2, 139 (1993).

Rueda, A. and Haisch, B., Physics Lett. A, 240, 115 (1998a). www.arxiv.org/abs/physics/9802031

Rueda, A. and Haisch, B., Found. Phys., 28, 1057 (1998b). www.arxiv.org/abs/physics/9802030

Rueda, A., Haisch B. and Tung, R., www.arxiv.org/gr-qc/0108026, (2001).

Schrödinger, E. Sitzungsb. Preuss. Akad. Wiss. Phys.-Math. K1. 24, 418 (1930); 3, 1 (1931).

Unruh, W. G., Phys. Rev. D 14, 870 (1976). 